Argonne

\title{
Status Assessment of the PROTEUS-ORIGEN Coupling
}

Nuclear Science and Engineering Division 


\title{
About Argonne National Laboratory
}

Argonne is a U.S. Department of Energy laboratory managed by UChicago Argonne, LLC under contract DE-AC02-06CH11357. The Laboratory's main facility is outside Chicago, at 9700 South Cass Avenue, Argonne, Illinois 60439. For information about Argonne and its pioneering science and technology programs, see www.anl.gov.

\section{DOCUMENT AVAILABILITY}

Online Access: U.S. Department of Energy (DOE) reports produced after 1991 and a growing number of pre-1991 documents are available free at OSTI.GOV (http://www.osti.gov/), a service of the US Dept. of Energy's Office of Scientific and Technical Information.

\author{
Reports not in digital format may be purchased by the public from the \\ National Technical Information Service (NTIS): \\ U.S. Department of Commerce \\ National Technical Information Service \\ 5301 Shawnee Rd \\ Alexandria, VA 22312 \\ www.ntis.gov \\ Phone: (800) 553-NTIS (6847) or (703) 605-6000 \\ Fax: (703) 605-6900 \\ Email: orders@ntis.gov
}

Reports not in digital format are available to DOE and DOE contractors from the Office of Scientific and Technical Information (OSTI):

U.S. Department of Energy

Office of Scientific and Technical Information

P.O. Box 62

Oak Ridge, TN 37831-0062

www.osti.gov

Phone: (865) 576-8401

Fax: (865) 576-5728

Email: reports@osti.gov

\section{Disclaimer}

This report was prepared as an account of work sponsored by an agency of the United States Government. Neither the United States Government nor any agency thereof, nor UChicago Argonne, LLC, nor any of their employees or officers, makes any warranty, express or implied, or assumes any legal liability or responsibility for the accuracy, completeness, or usefulness of any information, apparatus, product, or process disclosed, or represents that its use would not infringe privately owned rights. Reference herein to any specific commercial product, process, or service by trade name, trademark, manufacturer, or otherwise, does not necessarily constitute or imply its endorsement, recommendation, or favoring by the United States Government or any agency thereof. The views and opinions of document authors expressed herein do not necessarily state or reflect those of the United States Government or any agency thereof, Argonne National Laboratory, or UChicago Argonne, LLC. 


\section{Status Assessment of the PROTEUS-ORIGEN Coupling}

prepared by

S. M. Richards and E. R. Shemon

Nuclear Science and Engineering Division, Argonne National Laboratory

September 30, 2019 



\section{Introduction}

As part of the Nuclear Energy Advanced Modeling and Simulation (NEAMS) Campaign of the U.S. Department of Energy, Office of Nuclear Energy, an initiative was started to provide timedependent isotopic modeling capabilities to the PROTEUS neutronics module of the Simulationbased High-efficiency Advanced Reactor Prototyping (SHARP) toolkit. This capability is to be provided through a coupling with the Oak Ridge Isotope Generation (ORIGEN) code, which is part of the SCALE code package developed and maintained at Oak Ridge National Laboratory. This development has been a joint effort between the developers of PROTEUS at Argonne National Laboratory (ANL) and Oak Ridge National Laboratory (ORNL).

In general, the structuring of a coupled neutronics-depletion code works through iterative calculations and updates in time of the flux and isotopic concentrations in a problem. The steps of this process, in the simplest "predictor" model of depletion, are

1. The neutronics code calculates the multigroup scalar flux in a material region using provided initial concentrations and microscopic cross sections.

2. The flux calculated in step 1 and the initial material characteristics are then used to calculate the reaction rates in the material. The depletion code uses these reaction rates in the Bateman equations to calculate the changes in the isotopic concentrations in the material over a length of time, or time step.

3. These new concentrations are then used to recalculate the flux.

The current standard in depletion is the inclusion of a "corrector" step following the predictor step. The corrector step recalculates the reaction rates using the initial isotopic concentrations, but uses the flux calculated in step 3 at the end of the time step. The Bateman equations are then re-solved using this reaction rate and the "true" concentrations are taken to be the average of those found after the predictor step and those found after the corrector step. This correction accounts for the continuous nature of evolution of the material and the flux and the limits of the discrete treatment of it. The result is improvement in the accuracy of results and increased stability of the fluxisotopics solutions in time.

The purpose of this document is to assess the status of the PROTEUS-ORIGEN implementation. The project is known to be incomplete, but due to staffing changes and discontinued funding for collaborative work, it is essential to understand and document the current status.

\section{Coupling Structure}

The current implementation of the coupling of PROTEUS and ORIGEN only attempts the predictor model of coupled depletion. This is implemented through the driver file Depletion_SN2ND_Driver and calls to the first layer of the coupling interface Depletion_Import_Input, Depletion_Initialize_Solver, Depletion_Import_ASSIGNMENT, and 
Depletion_SN2ND_Solve. This layer handles the interfacing with the global data and the interactions between the PROTEUS-SN and the ORIGEN depletion architecture that is ultimately linked to ORIGEN through its automated programming interface (API).

The Depletion_Import_Input allocates and passes globally scoped input data containers to the DepletionAPI subroutines to read the user inputs and store the information in the data containers. In the next step in the coupling layer,Depletion_Initialize_Solver calls the Input_Depletion that was loaded in the previous step, and the Input_ISOTXS, and passes that global information to DepletionAPI via the DepletionAPI_Initialize subroutine. The DepletionAPI passes the loaded input information to the ORIGEN API DepletionAPI_ORIGEN via the DepletionAPI_Initialize_ORIGEN subroutine. The Origen API instantiates the basic definition constituents of an Origen library. The header is an object on a library that controls the interpolateable information for the library and controls a nuclide set that lists all of the nuclides tracked in that library. These nuclides are listed in the NuclideSet by ID and symbol, though ID (in SIZZZAAA form) is the only one used internally in ORIGEN to identify a nuclide. The mapping of PROTEUS isotope to ORIGEN naming scheme must be provided by hand by the user. The last of the set-up steps in the coupling prior to the solver implementation, Depletion_Import_ASSIGNMENT, connects the material information to mesh blocks as well as checks the isotope names and aliases, if one is given, against those known to ORIGEN and calculates and stores their "delay" (the number of transitions to arrive at an isotope from an initial material isotope) through Depletion_Check_Link. If a nuclide is given with no known equivalent in ORIGEN, an error will be thrown. In the solving step, the SN2ND calculations are set-up and performed in the same manner as takes place in other running modes. The reaction rates and scalar flux are then populated by $S N 2 N D \_E x p o r t \_D a t a$. This is the information, along with the initial material information, that is necessary to perform the depletion calculation. The depletion calculation is done through the ORIGEN Case structure using the library that was made in the initialization step. A new case is generated for each material block and if that block contains depletable materials, the block is depleted in serial using that block's properties. The depleted isotopic concentrations are then updated on that material. This process then repeats following the coupled depletion structure outlined previously. The results of these depletions are copied and transmitted, however, only total nuclide concentrations of O-16, U-235, and U-238 are in the output.

\section{Coupling Issues}

The current state of the coupling does not have all of the required functionality to be sufficient for the use within the SHARP toolkit. The main structural issue with the implementation is a lack of parallelization of the depletion. The solver currently is structured such that all material blocks are depleted, rather than only those blocks visible to a processor. In fact, at the initialization step in the coupling there is a check to see if the option to use spatial decomposition (parallelization in space) is enabled. If it is enabled, then the run is stopped as the current structure of the coupling does not support parallel depletion. The developers at ORNL have also reported that the current implementation does not update the one group cross sections from the SN2ND flux spectrum as the 
material depletes. This is due to them not being able pass the flux spectrum to the depletion solver and not being able to convert their cross section library to the ANL ISOTXS file format and not receiving one from ANL. This is a key requirement for accurate depletion, as the reaction rates at every subsequent time step will be incorrect. In its current state, only the initial depletion step for a problem run in serial would be expected to have the accuracy of the predictor implementation.

\section{Verification of Results in Current State}

In an attempt to verify the results given by the current state of the coupling, in the absence of any readily available benchmarks, a trivial problem was conceived to compare the results that are given by a hand calculation, a standalone ORIGEN calculation, a standalone PROTEUS calculation, and finally the coupled codes. This problem consists of U-235 and H-1 in an infinite homogenous medium. The 1 group nuclear data used is in table 1 , and was retrieved from an ORIGEN library that is used to dictate the transition structure in PROTEUS-ORIGEN. From this data and a hand calculation using the four-factor formula, an infinite multiplication factor of 1.2556 is expected from a material consisting of $10 \mathrm{~kg} \mathrm{U}-235$ and $30 \mathrm{~kg} \mathrm{H}-1$. This is in relative agreeance with the standalone PROTEUS-SN2ND calculation of 1.248 when a reflecting boundary is used for a 2-D 1x1 box with only the fuel region. PROTEUS-ORIGEN also results in an initial $\mathrm{k}$ value of 1.248 for this problem. However, standalone ORIGEN results in a predicted initial $\mathrm{k}_{\infty}$ of 1.088. While this is still critical, the discrepancy between this value and the other two are not currently understood.

\section{Table 1 Nuclear Data from library pwr.rev03.orglib}

\begin{tabular}{|c|c|c|c|}
\hline Nuclide & $\sigma_{a}(\mathrm{~b})$ & $\sigma_{F}(\mathrm{~b})$ & $\eta$ \\
\hline U-235 & 42.647 & 34.477 & 1.7879 \\
\hline H-1 & 0.025847 & 0.0 & 0.0 \\
\hline
\end{tabular}

The depletion problem was set in order to see a significant change in the U-235 concentration, as well as the $\mathrm{k}_{\infty}$. An arbitrary 100-day cycle length and a power in the fuel region of $9 \mathrm{MW}$ should result in approximately $9.47 \%$ burnup of the U-235 based on an average energy released per fission of $200 \mathrm{MeV}$. This calculation is closely matched by the standalone ORIGEN calculation where the initial mass of $10 \mathrm{~kg}$ is depleted to $8.82 \mathrm{~kg}$ and the $\mathrm{k}_{\infty}$. decreases from 1.088 to 0.998 . The discrepancy in this case to the hand calculation is the result of the cross sections changing as a function of burnup and the problem being broken into 1010 day steps as well as the energy generation calculation including all fissionable isotopes. However, with the same power, material, 
and depletion conditions in PROTEUS-ORIGEN results in an increasing $\mathrm{k}$ value from the initial 1.248 to 1.255 . The code also does not recognize the U-235 input material and does not output its depletion concentration. The option to output the full isotopic data as an output file was also not functioning at the time of the verification calculations. Due to this discrepancy, and not being able to check the depletion inventories, additional comparisons to the test problem were not possible.

Further "sanity" checks of the PROTEUS-ORIGEN depletion capabilities were also attempted using the supplied test cases. Based on the input power and initial mass of Uranium in the problems, a comparison of the fission cross sections to the total cross sections were done. This comparison is based on the total loss of U-235 vs. the expected loss of U-235 to fission and the input total cross sections and fission cross sections in the ANLXS files. This comparison should at least provide a bounding ratio as $\frac{\sigma_{f}}{\sigma_{t}}<\frac{\sigma_{f}}{\sigma_{a}}$, and the ratio of loss to fission and total absorption should be equivalent to $\frac{\sigma_{f}}{\sigma_{a}}$. However, the ratio of fission to absorption was significantly lower than the ratio of the cross sections. Due to this result and the lack of documentation for the inputs and outputs of the code, no conclusion can be made about the coupling's current state as there is no definitive proof that these errors are not the result of user error or misinterpretation.

\section{Recommendations}

Future work is necessary for the coupled depletion capabilities to be at the expected standard for this type of code. The first progress that needs to be made would be the correction of one group cross section updates in the serial implementation. Without this implemented, the code, even run in serial, will not give reliable results. The next most important work would be a proper parallel implementation of the depletion scheme. This work should be a concerted effort between the ANL and ORNL development team, so as to avoid the pitfalls and delays of past experiences. The current implementation disallowing spatial decomposition makes use of the code for any meaningful problems untenable. Finally, a predictor corrector scheme needs to be implemented to meet standard depletion practices for high-fidelity modeling capabilities like the SHARP toolkit. A demonstration of the envisioned structure and intended implementation of the coupling has been provided by the ORNL developers to guide further development efforts. However, given the ongoing difficulties from the differences in the PROTEUS and ORIGEN underlying structures, an alternative and potentially quicker route to implementing coupled depletion capabilities with PROTEUS is a native Bateman equation solver that is constructed from the beginning with the intention of using ISOTXS formatted nuclear data and the PROTEUS parallel architecture. 


\section{Argonne}

Nuclear Science and Engineering Division

Argonne National Laboratory

9700 South Cass Avenue, Bldg. 208

Argonne, IL 60439

www.anl.gov

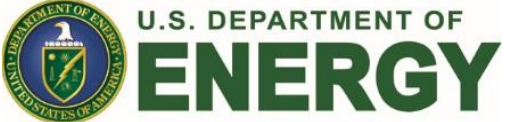

